\title{
Existencia de soluciones óptimas de programas matemáticos no lineales
}

\author{
Existence of optimal solutions of non-linear mathematical programs
}

\author{
${ }^{1}$ Jhony Alfonso Chávez Delgado, ${ }^{2}$ Hernan Enrique Lupaca Mamani
}

\begin{abstract}
RESUMEN
El propósito de este artículo es establecer condiciones para la existencia de soluciones óptimas de programas matemáticos no lineales dada la limitación que presenta el método de resolución gráfica cuando el número de variables de decisión es mayor que dos, entonces se dispuso de criterios más generales que garanticen la existencia de soluciones óptimas en programas matemáticos no lineales que surgen de forma inevitable en la aplicación a la ingeniería, tales como diseño de estructuras para la aviación y diseño de estructuras civiles. Se estableció que, si una función real de variable vectorial está definida en un conjunto de soluciones factibles cerrado y acotado, el programa no lineal es convexo. Así mismo, cuando el número de variables de decisión es mayor que dos la formulación y resolución computacional de programas matemáticos no lineales se resolvió utilizando el software Lingo. Se empleo en el desarrollo del trabajo de investigación el método lógico deductivo, apoyado en la teoría axiomática, para obtener condiciones sobre la existencia de soluciones óptimas en programas matemáticos no lineales. Finalmente se contrasto las demostraciones usando ejemplos conocidos de programas matemáticos no lineales y utilizando el software Lingo.
\end{abstract}

Palabras clave: programas matemáticos no lineales, existencia de soluciones óptimas, programación convexa, teorema de Weierstrass

\begin{abstract}
The purpose of this article is to establish conditions for the existence of optimal solutions of non-linear mathematical programs given the limitation of the graphic resolution method when the number of decision variables is greater than two, then there are general criteria that guarantee the existence of optimal solutions in non-linear mathematical programs that inevitably arise in the application to engineering, such as design of structures for aviation and design of civil structures. It was established that if a real vector variable function is defined in a set of closed and bounded feasible solutions, the nonlinear program is convex. Likewise, when the number of decision variables is greater than two, the formulation and computational resolution of nonlinear mathematical programs was solved using the software Lingo. The deductive logical method, based on axiomatic theory, was used in the development of research work to obtain conditions on the existence of optimal solutions in nonlinear mathematical programs. Finally, the demonstrations were contrasted using known examples of nonlinear mathematical programs and using the software Lingo.
\end{abstract}

Keywords: non-linear mathematical programs, existence of optimal solutions, convex programming, Weierstrass theorem

\footnotetext{
Universidad Nacional Jorge Basadre Grohmann. Tacna - Perú. E-mail: jchavezd@unjbg.edu.pe

2Universidad Nacional Jorge Basadre Grohmann. Tacna - Perú. E-mail: hlupaca29@gmail.com 


\section{INTRODUCCIÓN}

La programación matemática no lineal intenta dar respuesta a un tipo general de problemas donde se desea elegir el mejor entre un conjunto de elementos, en su forma más simple, el problema equivale a resolver una ecuación de este tipo

$$
\left\{\begin{array}{l}
\operatorname{Max}(\operatorname{Min}) f(x) \\
x \in S \subset{ }^{n} \\
x=\left(x_{1}, x,{ }_{2} \cdot ., n\right)
\end{array}\right.
$$

Algunas veces es posible expresar el conjunto de restricciones como solución de un sistema de igualdades o desigualdades

$$
\left\{\begin{array}{l}
g\left(x_{1}, x, \ldots, x_{n}\right)=0 \\
h\left(x_{1}, x, \ldots, x_{n}\right) \geq 0
\end{array}\right.
$$

Un problema de programación matemática no lineal trata de tomar una decisión óptima para maximizar (ganancia, velocidad, eficiencia, etc.) o minimizar en un criterio determinado (costo, tiempo, riesgo, error, etc.) las restricciones que significan que no cualquier decisión es posible.

Ante un problema óptimo parece razonable preguntarse en primer lugar sobre que variables podemos decidir. Una vez identificada las variables de decisión nos preguntamos cual es criterio que nos permita elegir la mejor decisión de acuerdo al objetivo que se pretende alcanzar. Es conveniente conocer cuáles son las distintas opciones disponibles, es decir, los valores posibles de las variables de decisión para que la solución de programas no lineales sea óptima. Estas condiciones es una generalización del método de los multiplicadores de Lagrange (1901).

Una vez expresado en términos matemáticos el problema que pretendemos resolver, nos preguntamos si tiene solución y en caso afirmativo, cuál es su localización. Cuando tenemos un programa matemático no lineal con dos variables de decisión su resolución gráfica puede darnos la respuesta. Dada la limitación que presenta el método de resolución gráfica cuando el número de variables de decisión es mayor que dos, conviene disponer de criterios generales que garanticen la existencia de soluciones óptimas de programas matemáticos no lineales. Los teoremas de Weierstrass (1894) y fundamental de la programación de la Programación convexa proporcionan condiciones suficientes de la existencia de óptimos globales y de globalidad de las soluciones de un programa matemático (Barbolla, 2001).

Este articulo contribuye a investigar las soluciones optimas de programas no lineales que ha sido estudiada por investigadores como Avriel (2003), Bertsekas (1999), Chiang (1987), Hiller (1967), Intriligator (1973), Nocedal (1999), Taha (1995) y otros.

\section{CONJUNTO CONVEXO Y FUNCIONES}

\section{Definición (Conjunto Convexo)}

Un subconjunto $C$ en $\square^{n}$ es convexo si y sólo si $\forall \bar{x}, \bar{y} \in C, \forall \lambda \in[0,1]$ se verifica

$$
\bar{z}=\lambda \bar{x}+(1-\lambda) \bar{y} \in C
$$

(Guerrero, 1997)

\section{Definición (Segmento cerrado)}

Se denomina segmento cerrado de extremos $\bar{x}, \bar{y}$ al conjunto

$$
[\bar{x}, \bar{y}]=\left\{\bar{z} \in \square^{n} / \bar{z}=\lambda \bar{x}+(1-\lambda) \bar{y}, \lambda \in[0,1]\right\}
$$

(Guerrero, 1997)

Definición (Segunda definición de Conjunto

Convexo) $C$ es convexo si y solo si

$\forall \lambda \in[0,1], \forall \bar{x}, \bar{y} \in C$ se tiene $[\bar{x}, \bar{y}] \subset C$.

(Guerrero, 1997)

\section{Definición (Combinación lineal)}

Dado un conjunto $M=\left\{\bar{x}_{1}, \bar{x}_{2}, \ldots, \bar{x}_{m}\right\}$ de elementos

$$
\begin{array}{lll}
\text { de } & \text { se } & \text { dice } \\
\bar{x}=\alpha_{1} \bar{x}_{1}+\alpha_{2} \bar{x}_{2}+\ldots+\alpha_{m} \bar{x}_{m}=\sum_{i=1}^{m} \alpha_{i} \bar{x}_{i}
\end{array}
$$

Es una combinación lineal de los elementos de $M$ si

$\alpha_{i} \in \square, i=\overline{1, m}$

(Guerrero, 1997)

\section{Definición (Combinación lineal no negativa)}

Dado un conjunto $M=\left\{\bar{x}_{1}, \bar{x}_{2} \ldots, \bar{x}_{m}\right\}$

, de elementos de $m$ se dice que $\bar{x}=\alpha_{1} \bar{x}_{1}+\alpha_{2} \bar{x}_{2}+\ldots+\alpha_{m} \bar{x}_{m}=\sum_{i=1}^{m} \alpha_{i} \bar{x}_{i}$ , es una combinación lineal no negativa de los elementos de $M$ si $\alpha_{i} \geq 0, i=\overline{1, m}$

(Guerrero, 1997)

\section{Definición (Combinación lineal convexa)}

Dado un conjunto $M=\left\{\bar{x}_{1}, \bar{x}_{2}, \ldots, \bar{x}_{m}\right\}$

, de elementos de $\square^{m}$ se dice que $\bar{x}=\alpha_{1} \bar{x}_{1}+\alpha_{2} \bar{x}_{2}+\ldots+\alpha_{m} \bar{x}_{m}=\sum_{i=1}^{m} \alpha_{i} \bar{x}_{i}$ , es una combinación lineal convexa de los elementos de $M$ si y solo si 
$\alpha_{i} \in[0,1], \sum_{i=1}^{m} \approx 1, i \quad \overline{1, m}=$

(Balbas, 1987)

Proposición (convexidad) Sean $X_{1}$ y $X_{2}$ dos conjuntos convexos de $\square^{n}$, se verifica que:

$X_{1} \cap X_{2}$ convexo

$X_{1}+X_{2}=\left\{\bar{x} \in \square^{2} / \exists x_{1} \in X_{1} x_{2} \in X_{2} ; \bar{x}=x_{1}+x_{2}\right\}$

convexo

(Balbas, 1987)

\section{Definición (funciones convexas)}

Sea $M$ un conjunto convexo y no vació de $\square^{n}$. La función $f: M \rightarrow \square$ es convexa si y solo si $\forall \bar{x}, \bar{y} \in M \forall \lambda \in[0,1]$ se cumple:

$f(\lambda \bar{x}+(1-\lambda) \bar{y}) \leq \lambda f(\bar{x})+(1-\lambda) f(\bar{y})$

(Apostol, 1973)

\section{Definición (funciones cóncavas)}

Sea $M$ un conjunto convexo y no vació de $\square^{n}$. La función $f: M \rightarrow \square$ es cóncava si y solo si $\forall \bar{x}, \bar{y} \in M \forall \lambda \in[0,1]$ se cumple: $f(\lambda \bar{x}+(1-\lambda) \bar{y}) \geq \lambda f(\bar{x})+(1-\lambda) f(\bar{y})$

(Apostol, 1973)

\section{Definición (funciones estrictamente convexas)}

Sea $M$ un conjunto convexo y no vació de $\square^{n}$. La función $f: M \rightarrow \square$ es estrictamente convexa si y sólo si $\forall \bar{x}, \bar{y} \in M$ con $\bar{x} \neq \bar{y}, \forall \lambda \in(0,1)$ se cumple:

$$
f(\lambda \bar{x}+(1-\lambda) \bar{y})<\lambda f(\bar{x})+(1-\lambda) f(\bar{y})
$$

(Apostol, 1973)

\section{Definición (funciones estrictamente cóncavas)}

Sea $M$ un conjunto convexo y no vació de $\square^{n}$. La función $f: M \rightarrow \square$ es estrictamente cóncava $\forall \bar{x}, \bar{y} \in M \quad$ con $\bar{x} \neq \bar{y}, \forall \lambda \in(0,1)$ se cumple: $f(\lambda \bar{x}+(1-\lambda) \bar{y})>\lambda f(\bar{x})+(1-\lambda) f(\bar{y})$ (Apostol, 1973)

\section{MATERIALES Y MÉTODOS}

Para nuestro artículo es considerado material de estudio los programas no lineales que admiten la siguiente formulación general:

$$
(P)\left\{\begin{array}{l}
\text { Opt } f\left(x_{1}, x_{2}, \ldots, x_{n}\right) \\
s . a \\
h_{1}\left(x_{1}, x_{2}, \ldots, x_{n}\right) \leq 0 \\
h_{2}\left(x_{1}, x_{2}, \ldots, x_{n}\right) \leq 0 \\
h_{m}\left(x_{1}, x_{2}, \ldots, x_{n}\right) \leq 0 \\
g_{1}\left(x_{1}, x_{2}, \ldots, x_{n}\right) \geq 0 \\
g_{2}\left(x_{1}, x_{2}, \ldots, x_{n}\right) \geq 0 \\
g_{k}\left(x_{1}, x_{2}, \ldots, x_{n}\right) \geq 0
\end{array}\right.
$$

En esta formulación

$f, h_{i}, g_{j}: \square^{n} \rightarrow \square, i=\overline{1, m}, j=\overline{1, k}$, son funciones con valores reales de las variables $x_{1}, x_{2}, \ldots, x_{n}$

Se empleó en el desarrollo del articulo el método lógico deductivo apoyado en la teoría axiomática para obtener condiciones sobre existencia de óptimos globales en programas matemáticos no lineales.

\section{RESULTADOS}

\section{Resolución Gráfica de Programas Matemáticos no lineales}

La resolución grafica de programas matemáticos no lineales en el plano admiten la siguiente formulación

$$
\text { general. }(P)\left\{\begin{array}{l}
\text { Opt } f\left(x_{1}, x\right)_{2} \\
s . a \\
h_{1}\left(x_{1}, x_{2}\right) \leq 0 \\
h_{2}\left(x_{1}, x_{2}\right) \leq 0 \\
g_{1}\left(x_{1}, x_{2}\right) \geq 0 \\
h_{2}\left(x, x_{2}\right) \geq 0
\end{array}\right.
$$

$$
\begin{aligned}
& \text { En esta formulación, } \\
& f, h_{i}, h_{j}: \square^{2} \rightarrow \square, i=\overline{1,2}, j=\overline{1,2}, \quad \text { son }
\end{aligned}
$$
funciones con valores reales de las variables $x_{1}, x_{2}$. 


\section{Condiciones de globalidad de programas matemáticos}

\section{Criterios de máximos y mínimos globales:}

Sea $f: B \subset \square^{n} \rightarrow \square$ una función continua en $B$, es cerrada y acotada. Entonces existen:

$\bar{x}^{*} \in B$ tal que: $f\left(\bar{x}^{*}\right) \leq f(\bar{x}), \forall \bar{x} \in B$

$\bar{x}^{\circ} \in B$ tal que: $f\left(\bar{x}^{\circ}\right) \geq f(\bar{x}), \forall \bar{x} \in B$

Es decir,

$\bar{x}^{*}$ es un mínimo global de $f$ en $B$

$\bar{x}^{\circ}$ es un máximo global de $f$ en $B$

\section{Programación convexa:}

Dado el programa convexo

$$
\left\{\begin{array}{l}
\operatorname{Min} f\left(x_{1}, x_{2}, \ldots, x_{n}\right) \\
\operatorname{s.a} \\
\bar{x}=\left(x_{1}, x_{2}, \ldots, x_{n}\right) \in B \subset \square^{n}
\end{array}\right.
$$

Se dice que:

Si $\bar{x} \in B$ es un mínimo local, entonces $\bar{x}$ es $]$ un mínimo global.

El conjunto de todos los mínimos del programa es un conjunto convexo. Para el problema de máximo se obtiene un resultado análogo sustituyendo el concepto de mínimo por el máximo.

\section{DISCUSIÓN}

Resolución Gráfica de Programas Matemáticos no lineales:

La resolución grafica de programas matemáticos no lineales admiten la siguiente formulación general.

Se dice que $\bar{x}^{*}$ es un mínimo local del programa si existe

$$
r>0 / f(\bar{x}) \geq f\left(\bar{x}^{*}\right), \forall \bar{x} \in \beta\left(\bar{x}{ }^{*}, r\right) \cap B
$$

\section{Definición (Conjuntos de Nivel)}

Sea la función $f: D \subset \square^{n} \rightarrow \square$, para cada $k \in \square$ se define el conjunto de nivel $k$ de $f$, es decir

$$
S_{k}=\{\bar{x} \in D / f(\bar{x})=k\}
$$

\section{Definición (Conjunto factible)}

Se define el conjunto factible de un programa como el conjunto de puntos $\bar{x} \in \square^{n}$, que verifican la restricción del programa, es decir,

$$
\begin{aligned}
& B=\left\{\bar{x} \in \square^{n} / h_{i}\left(x_{1}, \ldots, x\right)=0 ; i=\overline{1, n} ;\right. \\
& \left.g_{j}\left(x_{1}, \ldots, x_{k}\right) \leq 0, j=\overline{1, k}\right\}
\end{aligned}
$$

\section{Definición (Máximo y Mínimo global)}

Dado un programa matemático

$$
\left\{\begin{array}{l}
\text { Opt } f\left(x_{1}, x_{2}\right) \\
\bar{x}=\left(x_{1}, x_{2}\right) \in B \subset \square^{2}
\end{array}\right.
$$

Se dice que $\bar{x}^{*}$ es un máximo global del programa si se verifica

$$
f(\bar{x}) \leq f\left(\bar{x}^{*}\right), \forall \bar{x} \in B
$$

Se dice que $\bar{x}^{*}$ es un mínimo global del programa si se verifica $f(\bar{x}) \geq f\left(\bar{x}^{*}\right), \forall \bar{x} \in B$

Se dice que $\bar{x}^{*}$ es un máximo global estricto del programa si se verifica $f(\bar{x})<f\left(\bar{x}^{*}\right), \forall \bar{x} \in B$

Se dice que $\bar{x}^{*}$ es un mínimo global estricto del programa si se verifica $f(\bar{x})>f\left(\bar{x}^{*}\right), \forall \bar{x} \in B$

\section{Definición (Máximo y Mínimo local)}

Dado un programa matemático

$$
\left\{\begin{array}{l}
\text { Opt } f\left(x_{1}, x_{2}\right) \\
\bar{x}=\left(x_{1}, x_{2}\right) \in B \subset \square^{2}
\end{array}\right.
$$

Se dice que $\bar{x}^{*}$ es un máximo local del programa si existe $r>0 / f(\bar{x}) \leq f\left(\bar{x}^{*}\right), \forall \bar{x} \in \beta\left(\bar{x}^{*}, r\right) \cap B$

Se dice que $\bar{x}^{*}$ es un máximo local estricto del programa si existe

$$
\begin{aligned}
& r>0 / f(\bar{x})<f\left(\bar{x}^{*}\right), \forall \bar{x} \in \beta\left(\bar{x}^{*}, r\right) \cap B, \\
& \bar{x} \neq \bar{x}^{*}
\end{aligned}
$$

Se dice que $\bar{x}^{*}$ es un mínimo local estricto del programa si existe $r>0 / f(\bar{x})>f\left(\bar{x}^{*}\right), \forall \bar{x} \in \beta\left(\bar{x}{ }^{*} r\right) \cap B$, $\bar{x} \neq \bar{x}^{*}$

Ejemplo (óptimo global) Dados el programa matemático no lineal

$$
\left\{\begin{array}{l}
\text { Opt } 9-x_{1}^{2}-x_{2}^{2} \\
\text { s.a } \\
x_{1}+x_{2} \leq 3 \\
x_{1} \geq 0, x_{2} \geq 0
\end{array}\right.
$$

Se pide 
a) Resolverlo gráficamente

b) Comprobación con el programa LINGO

\section{Solución}

\section{Resolverlo gráficamente:}

a1) Dibujar las curvas de nivel de la función objetivo:

$$
\begin{aligned}
& S_{k}=\left\{\left(x_{1}, x_{2}\right) \in \square^{2} / f\left(x_{1}, x_{2}\right)=k\right\} \\
& =\left\{\left(x_{1}, x_{2}\right) \in \square^{2} / 9-x_{1}^{2}-x_{2}^{2}=k, k \in \square\right\} \\
& k=0 \rightarrow 9-x_{1}^{2}-x_{2}^{2}=0 \rightarrow x_{1}^{2}+x_{2}^{2}=9 \\
& k=1 \rightarrow 9-x_{1}^{2}-x_{2}^{2}=1 \rightarrow x_{1}^{2}+x_{2}^{2}=8 \\
& k=2 \rightarrow 9-x_{1}^{2}-x_{2}^{2}=2 \rightarrow x_{1}^{2}+x_{2}^{2}=7 \\
& k=9 \rightarrow 9-x_{1}^{2}-x_{2}^{2}=9 \rightarrow x_{1}^{2}+x_{2}^{2}=0
\end{aligned}
$$

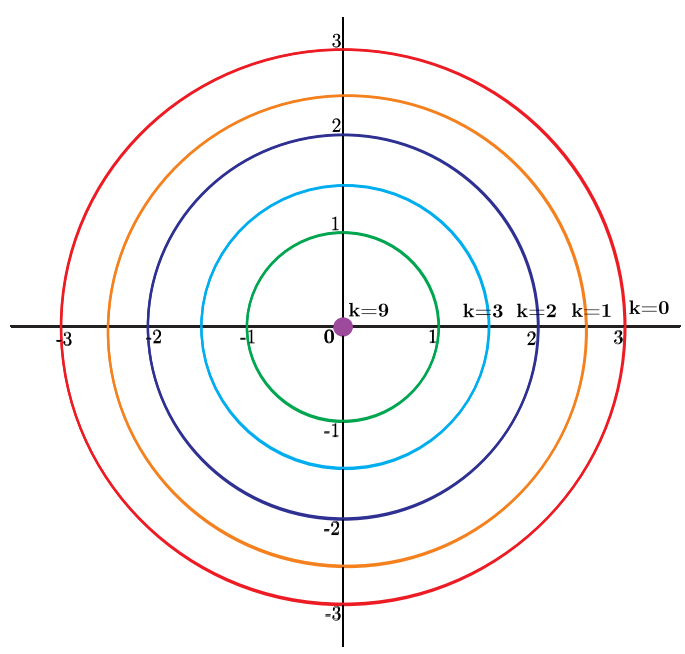

Figura 1. Conjunto de curvas de nivel

Fuente: Elaboración propia

Dibujar el conjunto de las soluciones factibles $B=\left\{\left(x_{1}, x_{2}\right) \in^{2} / x_{1}+x_{2} \leq 3, x_{1} \geq 0, x_{2} \geq 0\right\}$

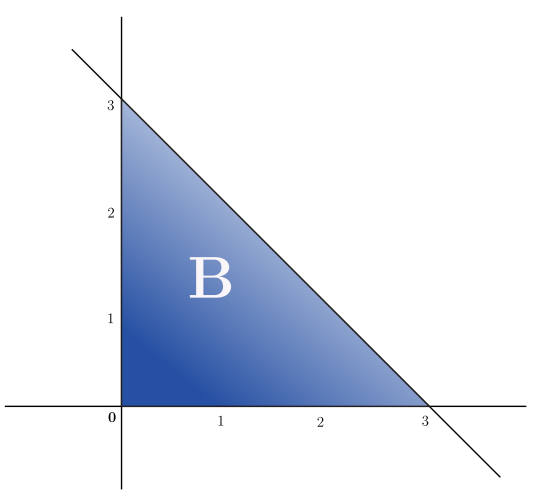

Figura 2. Conjunto de soluciones factibles Fuente: Elaboración propia

\section{Superposición de las figuras}

Figura 3. Superposición de figuras

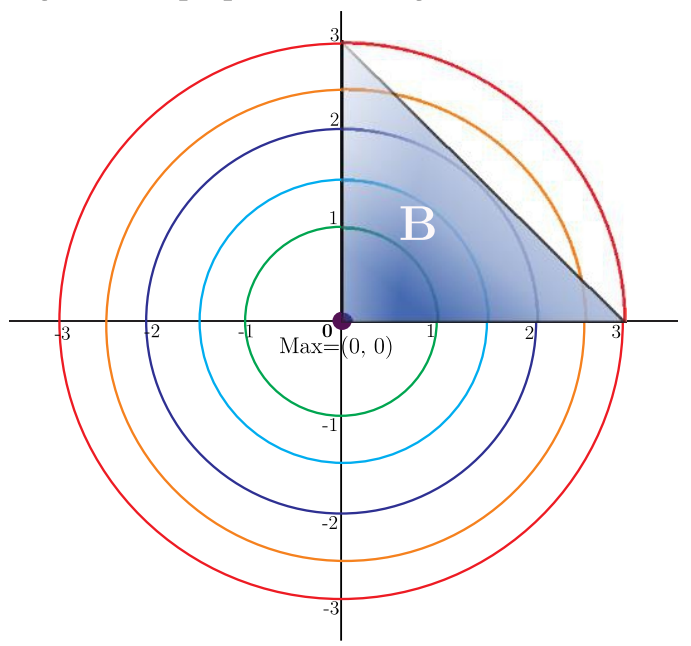

Fuente: Elaboración propia

En $\bar{x}^{*}=(0,0)$ existe un mínimo global y el valor de la función objetivo es $f(0,0)=9$.

\section{Comprobación con el software LINGO}

Tabla 1. Programa cuadrático

\begin{tabular}{|l|}
\hline \multicolumn{1}{|c|}{ PROGRAMA MATEMÁTICO NO } \\
LINEAL \\
MAX $=9-\mathrm{X} 1^{\wedge} 2-\mathrm{X} 2^{\wedge} 2 ;$ \\
¿SUJETO A LAS SIGUIENTES \\
RESTRICCIONES; \\
$\mathrm{X} 1+\mathrm{X} 2<=3 ;$ \\
$\mathrm{X} 1>=0 ;$ \\
$\mathrm{X} 2>=0 ;$ \\
\hline
\end{tabular}

Fuente: Elaboración propia con software LINGO. 
Tabla 2. Optimo global

\begin{tabular}{|c|c|c|}
\hline \multicolumn{3}{|c|}{ Global optimal solution found } \\
\hline Objetive value & \multicolumn{2}{|c|}{9.000000} \\
\hline Infeasibilities & \multicolumn{2}{|c|}{$0.1876777 \mathrm{E}-08$} \\
\hline $\begin{array}{ll}\text { Total } & \text { solver } \\
\text { iterations } & \\
\end{array}$ & \multicolumn{2}{|c|}{4} \\
\hline $\begin{array}{l}\text { Elapsed runtime } \\
\text { seconds }\end{array}$ & \multicolumn{2}{|c|}{0.19} \\
\hline \multicolumn{3}{|c|}{ Model is convex quadratic } \\
\hline Model Class & \multicolumn{2}{|c|}{ QP } \\
\hline Total variables & \multicolumn{2}{|c|}{2} \\
\hline Nonlinear variables & \multicolumn{2}{|c|}{2} \\
\hline Integer variables & \multicolumn{2}{|c|}{0} \\
\hline Total constraints & \multicolumn{2}{|c|}{4} \\
\hline $\begin{array}{l}\text { Nonlinear } \\
\text { constraints }\end{array}$ & \multicolumn{2}{|c|}{1} \\
\hline Total nonzeros & \multicolumn{2}{|c|}{6} \\
\hline Nonlinear nonzeros & \multicolumn{2}{|c|}{2} \\
\hline Variable & Value & $\begin{array}{l}\text { Reduced } \\
\text { Cost }\end{array}$ \\
\hline $\mathrm{X} 1$ & $\begin{array}{l}0.1954444 \mathrm{E}- \\
08\end{array}$ & $\begin{array}{l}0.4144295 \mathrm{E}- \\
08\end{array}$ \\
\hline $\mathrm{X} 2$ & $\begin{array}{l}0.1954444 \mathrm{E}- \\
08\end{array}$ & $\begin{array}{l}0.4144295 \mathrm{E}- \\
08\end{array}$ \\
\hline Row & $\begin{array}{l}\text { Slack or } \\
\text { Surplus }\end{array}$ & Dual Price \\
\hline 1 & 9.000000 & 1.000000 \\
\hline 2 & 3.000000 & 0.000000 \\
\hline 3 & $\begin{array}{l}0.1954444 \mathrm{E}- \\
08\end{array}$ & 0.000000 \\
\hline
\end{tabular}

1 conjunto de todos los mínimos del programa es un conjunto convexo. Para el problema de máximo se obtiene un resultado análogo sustituyendo el concepto de mínimo por el máximo.

\section{Prueba}

Fuente: Elaboración propia con software LINGO.

Condiciones de globalidad de programas matemáticos:

\section{Definición (Conjunto acotado)}

Un conjunto $C \subset \square^{m}$ es acotado si existe

$M>0 /\|x\|=\left(\sum_{i=1}^{n} x_{i}^{2}\right)^{1 / 2}<M, \forall \bar{x} \in C$

$\left(\sum_{i=1}^{n} x_{i}^{2}\right)^{1 / 2}=\sqrt{x_{1}^{2}+x_{2}^{2}+\ldots+t_{n} x^{2}}$

\section{Definición (Conjunto Abierto)}

Un conjunto $B \subset \square^{m}$ es abierto en $\square^{m}$, si y solo si $\forall \bar{x} \in B, \exists r>0$ tal que $B(\bar{x}, r) \subset B$

\section{Definición (Conjunto Cerrado)}

Un conjunto $B \subset \square^{m}$ es cerrado si su complemento es abierto en $\square^{m}$

\section{Criterio de Máximos y Mínimos Globales.} Teorema (Teorema de Weierstrass)

Sea $f: B \subset \square^{n} \rightarrow \square$ continua en $B, B$ es cerrado y acotado.

Entonces existen

$\bar{x}^{*} \in B$ tal que: $f\left(\bar{x}^{*}\right) \leq f(\bar{x}) ; \forall \bar{x} \in B$

$\bar{x}^{\circ} \in B$ tal que: $f\left(\bar{x}^{\circ}\right) \geq f(\bar{x}) ; \forall \bar{x} \in B$

Es decir:

$\bar{x}^{*}$ es un mínimo global de $f$ en $B$

$\bar{x}^{\circ}$ es un máximo global de $f$ en $B$

\section{Programación convexa}

Teorema (Teorema fundamental de la programación convexa)

Dado el programa convexo

$$
\left\{\begin{array}{l}
\operatorname{Min} f\left(x_{1}, x_{2}, \ldots, x_{n}\right) \\
\text { s.a. } \\
\bar{x}=\left(x_{1}, x_{2}, \ldots, x_{n}\right) \in B \subset \square^{n}
\end{array}\right.
$$

Se dice que:

Si $\bar{x} \in B$ es un mínimo local, entonces $\bar{x}$ es un mínimo global.

Supongamos que $\bar{x}_{1}$ es un mínimo local no global, entonces existe $\bar{x} \in B$ tal que $f(\bar{x})<f\left(\bar{x}_{1}\right)$.Como $B$ es convexo, para todo $\lambda \in[0,1]$, se verifica que

$$
\bar{z}=\lambda \bar{x}+(1-\lambda) \bar{x}_{1} \in B
$$

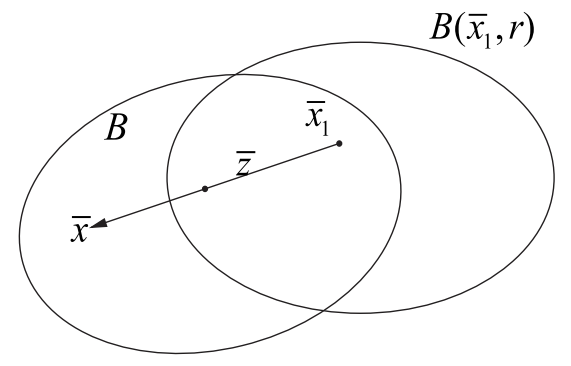

Figura 4. Conjunto convexo Fuente: Elaboración propia

Para cada $r>0$, existe $\lambda \in[0,1]$ tal que $\bar{z} \in B\left(\bar{x}_{1}, r\right) h B$ y por ser $f$ convexa se tiene $f(\bar{z})=f\left(\lambda \bar{x}+(1-\lambda) \bar{x}_{1}\right) \leq \lambda f(\bar{x})+(1-\lambda)<$ $<f\left(\bar{x}_{1}\right)+(1-\lambda) f\left(\bar{x}_{1}\right)=f\left(\bar{x}_{1}\right)$ 
, lo que contradice la hipótesis de que $\bar{x}_{1}$ es , un mínimo local del programa.

Sea $m$ el mínimo valor de $f$ en el conjunto $B \quad$ y sea $\quad S=\{\bar{x} \in B / f(\bar{x})=m\}$

Dados $\bar{x}, \bar{x}_{2} \in B \quad$ y $\lambda \in[0,1]$, se verifica que $\bar{z}=\lambda \bar{x}+(1-\lambda) \bar{x}_{2} \in S$ porque $m \leq f(\bar{z})=f\left(\lambda \bar{x}+(1-\lambda) \bar{x}_{2}\right) \leq$

$\leq \lambda f(\bar{x})+(1 \lambda) f\left(\bar{x}_{2}\right)=m$

, entonces $f(\bar{z})=m$

\section{Ejemplo (óptimo local)}

Dado el programa

$$
\left\{\begin{array}{l}
\text { Opt }\left(x_{1}-1\right)^{2}+\left(x_{2} x-2^{2}\right) \\
\text { s.a } \\
-x_{1}+x_{2} \leq 1 \\
x_{1}+x_{2} \leq 2 \\
x_{1} \geq 0, x_{2} \geq 0
\end{array}\right.
$$

Se pide:

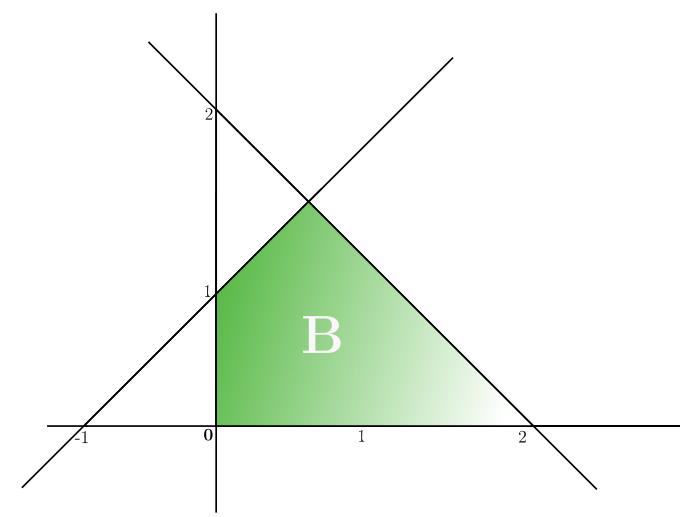

Figura 6. Conjunto de soluciones factibles

\section{Resolverlo gráficamente}

Estudiar analíticamente si verifica el teorema de Weierstrass

\section{Comprobar con el software LINGO}

\section{Solución:}

Gráficamente:

a1) Curvas de nivel de la función objetivo:

$$
\begin{aligned}
& S_{k}=\left\{\left(x_{1}, x_{2}\right) \in \square^{2} / f\left(x_{1}, x_{2}\right)=k\right\} \\
& \quad=\left\{\left(x_{1}, x_{2}\right) \in \square^{2} /\left(x_{1}-1\right)^{2}+\left(x_{2}-2\right)^{2}=k, k \in \square\right\} \\
& k<0 \rightarrow S_{k}=\varnothing
\end{aligned}
$$

$$
\begin{aligned}
& k=0 \rightarrow\left(x_{1}-1\right)^{2}+(x-2)^{2}=0 \\
& k=1 \rightarrow\left(x_{1}-1\right)^{2}+(x-2)^{2}=1 \\
& k=2 \rightarrow\left(x_{1}-1\right)^{2}+(x-2)^{2}=2 \\
& k=5 \rightarrow\left(x_{1}-1\right)^{2}+(x-2)^{2}=5
\end{aligned}
$$

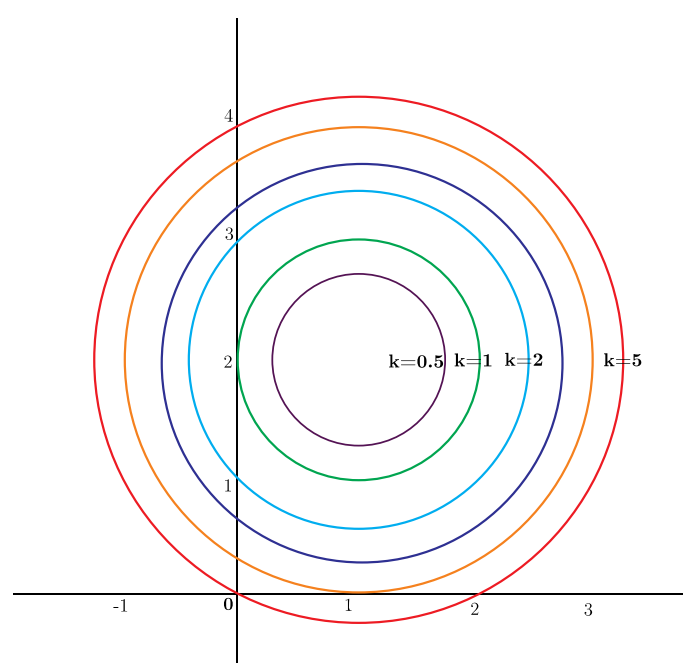

Figura 5. Conjunto de curvas de nivel Fuente: Elaboración propia

a2) Dibujo del conjunto de las soluciones factibles $B=\left\{\left(x_{1}, x_{2}\right) \in^{2} /-x_{1}+x_{2} \leq 1, x_{1}+x_{2} \leq 2, x_{1} \geq 0, x_{2} 0 \geq\right\}$

\section{Fuente: Elaboración propia}

a3) Superposición de las figuras

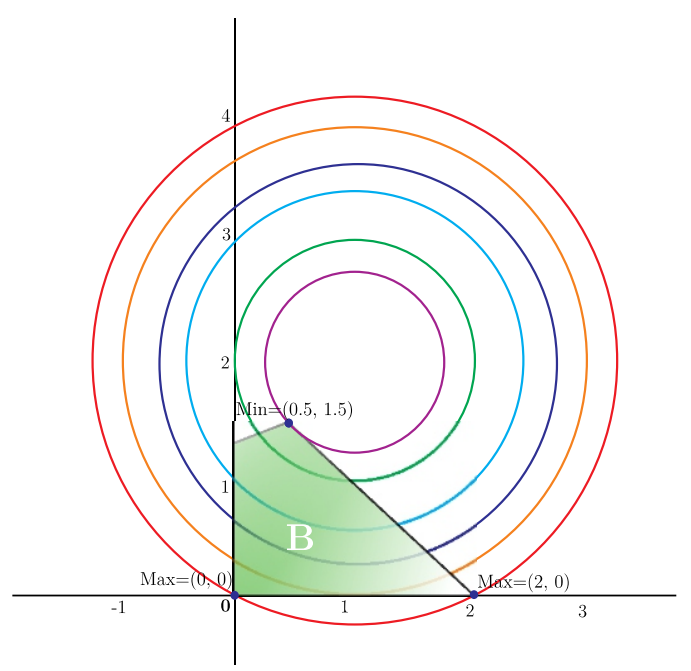

Figura 7. Superposición de figuras Fuente: Elaboración propia

Existe un mínimo global en la intersección de las rectas $-x_{1}+x_{2}=1$ y $x_{1}+x_{2}=2$ 
Entonces, $x_{1}=\frac{1}{2}, x_{2}=\frac{3}{2}$

Luego en $\bar{x}^{*}=\left(\frac{1}{2}, \frac{3}{2}\right)$ existe un mínimo global y el valor de la función objetivo es $f\left(\frac{1}{2}, \frac{3}{2}\right)=\left(\frac{1}{2}\right)$

En los puntos $\bar{x}^{*}=(0,0)$ y $\bar{x}^{*}=(2,0)$ existen máximos globales $y$ el valor de la función es $f(0,0) \neq(2,0 \neq 5$.

\section{Analíticamente:}

Veamos que en este caso si cumple las tres hipótesis del teorema (Weierstrass)

b1) $f$ debe ser continua

En efecto, $f\left(x_{1}, x_{2}\right)=\left(x_{1}-1\right)^{2}+\left(x_{2}-2\right)^{2}$ es continua por ser una función polinómica.

b2) $B$ es cerrado

En efecto, $\quad g_{1}\left(x_{1}, x_{2}\right)=-x_{1}+x_{2}$,

$g_{2}\left(x_{1}, x_{2}\right)=x_{1}+x_{2}, g_{3}\left(x_{1}, x_{2}\right)=x_{1}, g_{4}\left(x_{1}, x_{2}\right)=x_{2}$

, son funciones continuas, entonces

$g_{1}^{-1}\{(-\infty, 1]\}=\left\{\left(x_{1}, x_{2}\right) \in \square^{2} \quad /-x_{1}+x_{2} \leq 1\right\}$

, cerrado

$g_{2}^{-1}\{(-\infty, 2]\}=\left\{\left(x_{1}, x_{2}\right) \in \square^{2} \quad / x_{1}+x_{2} \leq 2\right\}$

, cerrado

$g_{3}^{+}\{[0,+\infty)\}=\left\{\left(x_{1}, x_{2}\right) \in \square^{2} / x_{1} \geq 0\right\}$

cerrado

$g_{4}^{+}\{[0,+\infty)\}=\left\{\left(x_{1}, x_{2}\right) \in \square^{2} / x_{2} \geq 0\right\}$

cerrado

b3) $B$ es un conjunto acotado porque $\exists M=\sqrt{5}>0 /\|\bar{x}\|=\sqrt{x_{1}^{2}+x_{2}^{2}} \leq \sqrt{5}, \forall \bar{x} \in B$.

Como se cumplen las tres condiciones del teorema de Weierstrass, el programa dado tiene máximo y mínimo global.

\section{c) Comprobación con el software LINGO}

Tabla 3. Programa cuadrático

PROGRAMA MATEMÁTICO NO LINEAL

MAX $=(X 1-1)^{\wedge} 2+(X 2-2)^{\wedge} 2$

\section{¡SUJETO A LAS SIGUIENTES}

RESTRICCIONES;

$-\mathrm{X} 1+\mathrm{X} 2<=1 ;$

$\mathrm{X} 1+\mathrm{X} 2<=2$

$\mathrm{X} 1>=0$

$\mathrm{X} 2>=0$;

Fuente: Elaboración propia con software LINGO.

Tabla 4. óptimo local

\begin{tabular}{|c|c|c|}
\hline \multicolumn{3}{|c|}{ Local optimal solution found } \\
\hline Objetive value & \multicolumn{2}{|c|}{5.000000} \\
\hline Infeasibilities & \multicolumn{2}{|c|}{0.000000} \\
\hline $\begin{array}{ll}\text { Total } & \text { solver } \\
\text { iterations } & \end{array}$ & \multicolumn{2}{|c|}{22} \\
\hline $\begin{array}{ll}\text { Elapsed } & \text { runtime } \\
\text { seconds } & \end{array}$ & \multicolumn{2}{|c|}{1.25} \\
\hline \multicolumn{3}{|c|}{ Model is convex quadratic } \\
\hline Model Class & \multicolumn{2}{|c|}{ QP } \\
\hline Total variables & \multicolumn{2}{|c|}{2} \\
\hline Nonlinear variables & \multicolumn{2}{|c|}{2} \\
\hline Integer variables & \multicolumn{2}{|c|}{0} \\
\hline Total constraints & \multicolumn{2}{|c|}{5} \\
\hline Nonlinear constraints & \multicolumn{2}{|c|}{1} \\
\hline Total nonzeros & \multicolumn{2}{|c|}{8} \\
\hline Nonlinear nonzeros & \multicolumn{2}{|c|}{2} \\
\hline Variable & Value & $\begin{array}{l}\text { Reduced } \\
\text { Cost }\end{array}$ \\
\hline $\mathrm{X} 1$ & 0.000000 & 2.000000 \\
\hline $\mathrm{X} 2$ & 0.000000 & 4.000000 \\
\hline Row & $\begin{array}{l}\text { Slack or } \\
\text { Surplus }\end{array}$ & Dual Price \\
\hline 1 & 5.000000 & 1.000000 \\
\hline 2 & 1.000000 & 0.000000 \\
\hline 3 & 2.000000 & 0.000000 \\
\hline 4 & 0.000000 & 0.000000 \\
\hline 5 & 0.000000 & 0.000000 \\
\hline
\end{tabular}

Fuente: Elaboración propia con software LINGO.

\section{CONCLUSIONES}

El teorema de Weierstrass nos da la existencia de máximos y mínimos globales en el estudio de la resolución de programas matemáticos no lineales.

El teorema de la Programación convexa nos da la convexidad del programa en el que todo óptimo local sea global. 


\section{REFERENCIAS BIBLIOGRÁFICAS}

APOSTOL, T. Calculus, Vols. I yII, 2nd Edition, Reverté, 1973.

AVRIEL, Mordecai. Nonlinear Programming: Analysis and Methods. Dover Publishing.2003

BERNOULLI, J. y Leibniz, G. W. Philosophicum et mathematicum. 1745

BERTSEKAS, D. P. Nonlinear Programming: 2nd Edition. Athena. Scientific.1999

BALBAS, A y Gil, J. A. Programación matematica, Editorial AC.1987

BARBOLLA, Rosa. Optimización. PEARSON EDUCACIÓN, S.A, Madrid 2001

CHIANG, A. Metodos fundamentals de economia matemática, 3raedición, McGraw Hill.1987
DANTZIG. Linear Programming and extensions, Princeton ,1963

GUERRERO Casas, F. Curso de Optimización: Programación matemática, Ariel economía.1997

HILLER F. y Lieberman G. Introducción a la Investigación de Operaciones, México: Mc Graw Hill.1967

INTRILIGATOR, M. Optimización matemática y teoria economica, Prentice-Hall.1973

LAGRANGE, J. L. Elementary Mathematics, Chicago, 1901

NOCEDAL, J. and Wright, S. J. Numerical Optimization. Springer.1999

TAHA H. Investigación de Operaciones, México: Pearson 1995

WEIERSTRASS, K. Mathematische werke, Berlin, 1894 